\title{
Practical Designs of Brain-Computer Interfaces Based on the Modulation of EEG Rhythms
}

\author{
Yijun Wang, Xiaorong Gao, Bo Hong, and Shangkai Gao
}

\section{Introduction}

A brain-computer interface (BCI) is a communication channel which does not depend on the brain's normal output pathways of peripheral nerves and muscles [1-3]. It supplies paralyzed patients with a new approach to communicate with the environment. Among various brain monitoring methods employed in current BCI research, electroencephalogram (EEG) is the main interest due to its advantages of low cost, convenient operation and non-invasiveness. In present-day EEG-based BCIs, the following signals have been paid much attention: visual evoked potential (VEP), sensorimotor mu/beta rhythms, P300 evoked potential, slow cortical potential (SCP), and movement-related cortical potential (MRCP). Details about these signals can be found in chapter "Brain Signals for Brain-Computer Interfaces". These systems offer some practical solutions (e.g., cursor movement and word processing) for patients with motor disabilities.

In this chapter, practical designs of several BCIs developed in Tsinghua University will be introduced. First of all, we will propose the paradigm of BCIs based on the modulation of EEG rhythms and challenges confronting practical system designs. In Sect. 2, modulation and demodulation methods of EEG rhythms will be further explained. Furthermore, practical designs of a VEP-based BCI and a motor imagery based BCI will be described in Sect. 3. Finally, Sect. 4 will present some real-life application demos using these practical BCI systems.

\subsection{BCIs Based on the Modulation of Brain Rhythms}

Many of the current BCI systems are designed based on the modulation of brain rhythms. For example, power modulation of mu/beta rhythms is used in the BCI system based on motor imagery [4]. Besides, phase modulation is another method

\footnotetext{
S. Gao $(\bowtie)$

Department of Biomedical Engineering, School of Medicine, Tsinghua University, Beijing, China e-mail: gsk-dea@tsinghua.edu.cn
} 
which has been employed in a steady-state visual evoked potential (SSVEP) based BCI [5]. More generally, evoked potentials can be considered to result partially from a reorganization of the phases of the ongoing EEG rhythms [6].

From the viewpoint of psychophysiology, EEG signals are divided into five rhythms in different frequency bands: delta rhythm $(0.1-3.5 \mathrm{~Hz})$, theta rhythm (4-7.5 Hz), alpha rhythm $(8-13 \mathrm{~Hz})$, beta rhythm $(14-30 \mathrm{~Hz})$, and gamma rhythm $(>30 \mathrm{~Hz})$ [7]. Although the rhythmic character of EEG has been observed for a long period, many new studies on the mechanisms of brain rhythms emerged after the 1980s. So far, the cellular bases of EEG rhythms are still under investigation. The knowledge of EEG rhythms is limited; however, numerous neurophysiologic studies indicate that brain rhythms can reflect changes of brain states caused by stimuli from the environment or cognitive activities. For example, EEG rhythms can indicate working state or idling state of the functional areas of the cortex. It is known that the alpha rhythm recorded over the visual cortex is considered to be an indicator of activities in the visual cortex. The clear alpha wave while eyes are closed indicates the idling state of the visual cortex, while the block of the alpha rhythm when eyes are open reflects the working state of the visual cortex. Another example is mu rhythm, which can be recorded over the sensorimotor cortex. A significant mu rhythm only exists during the idling state of the sensorimotor cortex. The block of mu rhythm accompanies activation of the sensorimotor cortex.

Self control of brain rhythms serves an important function in brain-computer communication. Modulation of brain rhythms is used here to describe the detectable changes of EEG rhythms. BCIs based on the modulation of brain rhythms recognize changes of specific EEG rhythms induced by self-controlled brain activities. In our studies, we focus on EEG rhythms located in the frequency band of alpha and beta rhythms $(8-30 \mathrm{~Hz})$. Compared with the low-frequency evoked potentials, these components have several advantages as follows:

(1) Affected less by artifacts. The lower frequency components are always easily affected by the artifacts introduced by electrooculogram (EOG) and electromyogram (EMG). For example, removal of the eye movement artifact is an important procedure in event-related potential (ERP) processing, whereas it can be omitted in analyzing mu/beta rhythms after specific band-pass filtering.

(2) More stable and lasting, and thus with a higher signal-to-noise ratio (SNR). Modulation of these rhythms can last for a relatively long period (e.g., several seconds), while transient evoked potentials only occur within several $100 \mathrm{~ms}$. Therefore, it is more promising to realize single-trial identification for highfrequency brain rhythms. Moreover, due to the phase-locked character of the evoked potentials, strict time precision is necessary for data alignment in the averaging procedure, while analysis of the power-based changes of the highfrequency rhythms permits a lower precision, and thus facilitates online designs of hardware and software.

(3) More applicable signal processing techniques. For wave-based analysis of evoked potentials, analysis is always done in the temporal domain. However, for the high-frequency rhythms, various temporal and frequency methods (e.g., 
Brain-Computer Interface

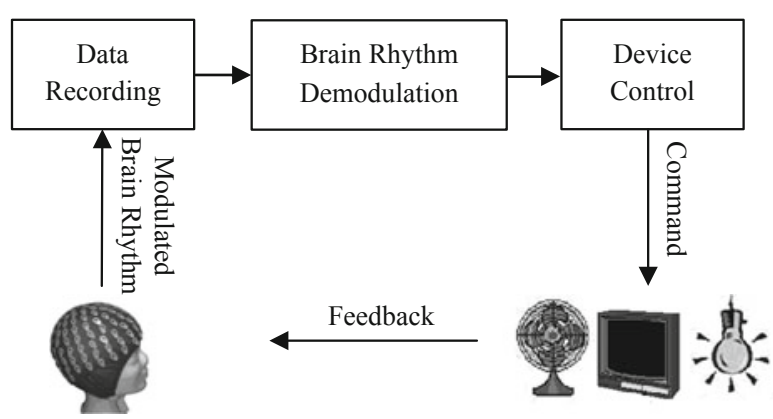

Fig. 1 Diagram of a BCI based on the modulation of brain rhythms

power and coherence analysis methods in the temporal and frequency domains, respectively) can be applied to extract EEG features. Therefore, the BCI based on the high-frequency rhythms provides a better platform for studying signal processing techniques and has a bigger potential in system performance.

Figure 1 is the block diagram of a BCI based on the modulation of brain rhythms. The control intention of the subject is embedded in the modulated brain rhythms through specific approaches, e.g., frequency coding or phase coding. According to the modulation approaches, the signal processing procedure aims to demodulate the brain rhythms and then extract the features, which will be translated into control signals to operate the output device. For instance, the SSVEP-based BCI and the motor imagery based BCI adopt typical approaches of brain rhythm modulation. The SSVEP system uses detection of frequency-coded or phase-coded SSVEPs to determine the gaze or spatial selective attention direction of the subject. Unlike the SSVEP BCI, the motor imagery BCI recognizes spatial distributions of amplitudemodulated mu/beta rhythms corresponding to the motor imagery states of different body parts. The methods for demodulating brain rhythms applied in BCIs will be introduced in the next section.

\subsection{Challenges Confronting Practical System Designs}

The design and implementation of an online system plays an important role in current BCI research, with the purpose of producing practical devices for real-life clinical application [8]. Compared with offline data analysis, an online BCI has difficulties in fulfilling real-time processing, system practicability and brain-machine co-adaptation. In this chapter, the principle of an online BCI based on the rhythmic modulation of EEG signals will be proposed. In our studies, two rhythmic EEG components corresponding to brain activities from the sensorimotor cortex and the visual cortex, i.e., mu rhythm and SSVEP, have been investigated and employed in constructing different types of online BCI systems. 
After many studies carried out to implement and evaluate demonstration systems in laboratory settings, the challenge facing the development of practical BCI systems for real-life applications needs to be emphasized. There is still a long way to go before BCI systems can be put into practical use. The feasibility for practical application is a serious challenge in the current study. To design a practical BCI product, the following issues need to be addressed.

(1) Convenient and comfortable to use. Current EEG recording systems use standard wet electrodes, in which electrolytic gel is required to reduce electrodeskin interface impedance. Using electrolytic gel is uncomfortable and inconvenient, especially when a large number of electrodes are adopted. An electrode cap with a large numbers of electrodes is uncomfortable for users to wear and thus unsuitable for long-term recording. Besides, the preparation for EEG recording takes a long time, thus making BCI operation boring. Moreover, recording hardware with a large amount of channels is quite expensive, so that it is difficult for common users to afford. For these reasons, reducing the number of electrodes in BCI systems is a critical issue for the successful development of clinical applications of BCI technology.

(2) Stable system performance. Considering data recording in unshielded environments with strong electromagnetic interference, employment of an active electrode may be much better than a passive electrode. It can ensure that the recorded signal is insensitive to interference [9]. During system operation, to reduce the dependence on technical assistance, ad hoc functions should be provided in the system to adapt to non-stationarity of the signal caused by changes of electrode impedance or brain state. For example, software should be able to detect bad electrode contact in real time and automatically adjust algorithms to be suitable for the remaining good channels.

(3) Low-cost hardware. Most BCI users belong to the disabled persons' community; therefore, the system can not be popularized if it costs too much, no matter how good its performance is. When considering the following aspects, reducing system costs while maintaining performance might be expected. On one hand, to reduce the cost of commercial EEG equipment, a portable EEG recording system should be designed just to satisfy the requirement of recording the specific brain rhythms. On the other hand, to eliminate the cost of a computer used for signal processing in most current BCIs, a digital signal processor (DSP) should be employed to construct a system without dependency on a computer.

\section{Modulation and Demodulation Methods for Brain Rhythms}

In rhythm modulation-based BCIs, the input of a BCI system is the modulated brain rhythms with embedded control intentions. Brain rhythm modulation is realized by executing task-related activities, e.g., attending to one of several visual stimuli. Demodulation of brain rhythms can extract the embedded information, which will 
be converted into a control signal. The brain rhythm modulations could be sorted into the following three classes: power modulation, frequency modulation, and phase modulation. For a signal $s(t)$, its analytical signal $z(t)$ is a complex function defined as:

$$
z(t)=s(t)+j \hat{s}(t)=A(t) e^{j \phi(t)}
$$

where $\hat{s}(t)$ is the Hilbert transform of $s(t), A(t)$ is the envelope of the signal, and $\phi(t)$ is the instantaneous phase. Changes of the modulated brain rhythms can be reflected by $A(t)$ or $\phi(t)$. In this section, three examples will be introduced to present the modulation and demodulation methods for mu rhythm and SSVEPs. More information about BCI related signal processing methods can be found in chapter "Digital Signal Processing and Machine Learning" of this book.

\subsection{Power Modulation/Demodulation of Mu Rhythm}

BCI systems based on classifying single-trial EEGs during motor imagery have developed rapidly in recent years $[4,10,11]$. The physiological studies on motor imagery indicate that EEG power differs between different imagined movements in the motor cortex. The event-related power change of brain rhythms in a specific frequency band is well known as event-related desynchronization and synchronization (ERD/ERS) [12]. During motor imagery, mu (8-12 Hz) and beta (18-26 Hz) rhythms display specific areas of ERD/ERS corresponding to each imagery state. Therefore, motor imagery can be performed to implement a BCI based on the power modulation of mu rhythms.

The information coding by power modulation is reflected by $A(t)$ in (1). The features extracted from the modulated mu rhythms for further recognition of the $N$-class motor imagery states can be defined as:

$$
f_{i}^{\text {Power }}(t)=A_{i}(t), i=1,2, \ldots N
$$

The method by means of the analytical signal presents a theoretical description of EEG power modulation and demodulation. In real systems, demodulation is usually realized through estimating power spectral density (PSD) [13]. In online systems, to reduce computational cost, a practical approach is to calculate band-pass power in the temporal domain, i.e., calculating the mean value of the power samples derived from squaring the amplitude samples. Figure 2 shows three single-trial EEG waveforms and PSDs over the left motor cortex (electrode C3), corresponding to imaginations of left/right hand and foot movements for one subject. PSD was estimated by the periodogram algorithm, which can be easily executed by fast Fourier transform (FFT). Compared to the state of left-hand imagination, right-hand imagination induces an obvious ERD (i.e., power decrease), while foot imagination results in a significant ERS characterized as a power increase. 


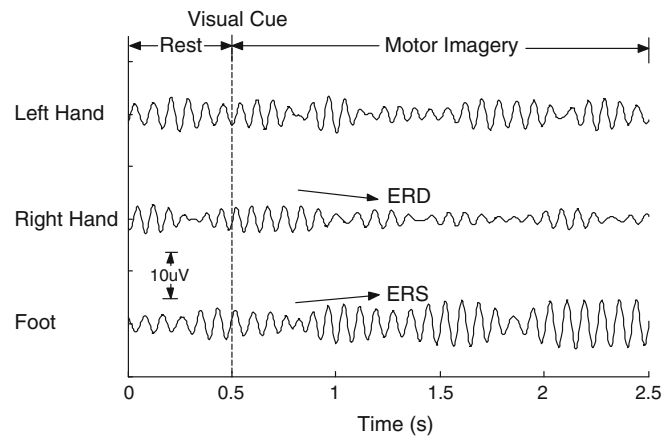

(a)

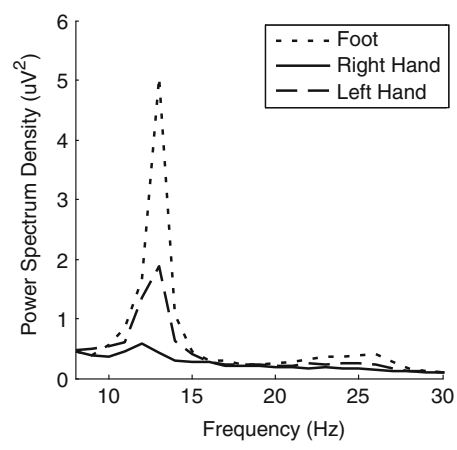

(b)

Fig. 2 (a) Examples of single-trial waveforms of mu rhythms on electrode C3 during three different motor imagery tasks. Data were band-pass filtered at $10-15 \mathrm{~Hz}$. A visual cue for starting motor imagery appears at $0.5 \mathrm{~s}$, indicated by the dotted line. On electrode C3, ERD and ERS of mu rhythms correspond to imagination of right hand and foot movements, respectively. (b) Averaged PSDs on electrode C 3 corresponding to three different motor imagery tasks

\subsection{Frequency Modulation/Demodulation of SSVEPS}

The BCI system based on VEPs has been studied since the 1970s [14]. Studies on the VEP BCI demonstrate convincing robustness of system performance through many laboratory and clinical tests [15-21]. The recognized advantages of this BCI include easy system configuration, little user training, and a high information transfer rate (ITR). VEPs are derived from the brain's response to visual stimulation. SSVEP is a response to a visual stimulus modulated at a frequency higher than $6 \mathrm{~Hz}$. The photic driving response, which is characterized by an increase in amplitude at the stimulus frequency, results in significant fundamental and second harmonics. The amplitude of SSVEP increases enormously as the stimulus is moved closer to the central visual field. Therefore, different SSVEPs can be produced by looking directly at one of a number of frequency-coded stimuli. Frequency modulation is the basic principle of the BCIs using SSVEPs to detect gaze direction.

$s(t)$ in (1) is supposed to be the frequency-coded SSVEP, and the feature used for identifying the fixed visual target is the instantaneous frequency, which can be calculated as:

$$
f_{i}^{\text {Frequency }}(t)=\frac{d \phi_{i}(t)}{d t}, i=1,2, \ldots, N
$$

where $\phi(t)$ is the instantaneous phase, and $N$ is the number of visual targets. Ideally, the instantaneous frequency of the SSVEP should be in accord with the stimulation frequency. In a real system, frequency recognition adopts the approach of detecting the peak value in the power spectrum. The visual target will induce a peak in the amplitude spectrum at the stimulus frequency. For most subjects, the amplitudes in the frequency bands on both sides will be depressed, thus facilitating peak detection. 


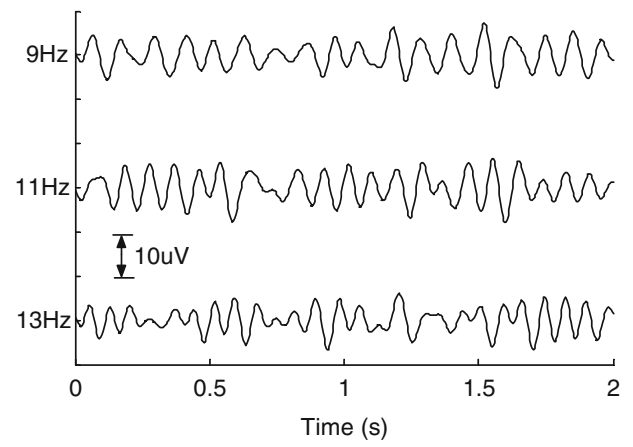

(a)

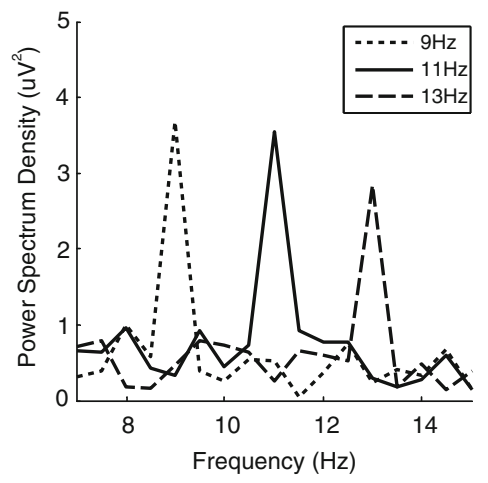

(b)

Fig. 3 (a) Examples of single-trial SSVEP waveforms over the occipital region. Data were bandpass filtered at 7-15 Hz. The stimulation frequencies were 9, 11, and $13 \mathrm{~Hz}$. (b) PSDs of the three single-trial SSVEPs. The stimulation frequency is clearly shown at the peak value of the PSD

Demodulation of a frequency-coded SSVEP is to search for the peak value in the power spectrum and determine the corresponding frequency. Figure 3 shows three waveforms of frequency-coded SSVEPs (at $9 \mathrm{~Hz}, 11 \mathrm{~Hz}$, and $13 \mathrm{~Hz}$, respectively) and their corresponding power spectra. The target can be easily identified through peak detection in the power spectrum.

\subsection{Phase Modulation/Demodulation of SSVEPS}

In the SSVEP-BCI system based on frequency modulation, flickering frequencies of the visual targets are different. In order to ensure a high classification accuracy, a sufficient frequency interval should be kept between two adjacent stimuli and the number of targets will then be restricted. If phase information embedded in SSVEP can be added, the number of flickering targets may be increased and a higher ITR can be expected. An SSVEP BCI based on phase coherent detection was first proposed in [5], and its effectiveness was confirmed. However, only two stimuli with the same frequency but different phases were dealt with in their design, and the advantages of phase detection were not sufficiently shown. Inspired by their work, we tried to further the work by designing a system with stimulating signals of six different phases under the same frequency.

The phase-coded SSVEP can ideally be considered a sine wave at a frequency similar to the stimulation frequency. Suppose $s(t)$ in (1) is the phase-coded SSVEP, the feature used for identifying the fixed visual target is the instantaneous phase:

$$
f_{i}^{\text {Phase }}(t)=\phi_{i}(t), i=1,2, \ldots N
$$

The first step of implementing a phase-encoded SSVEP BCI is its stimuli design. Spots flickering on a computer screen at the same frequency with strictly constant 


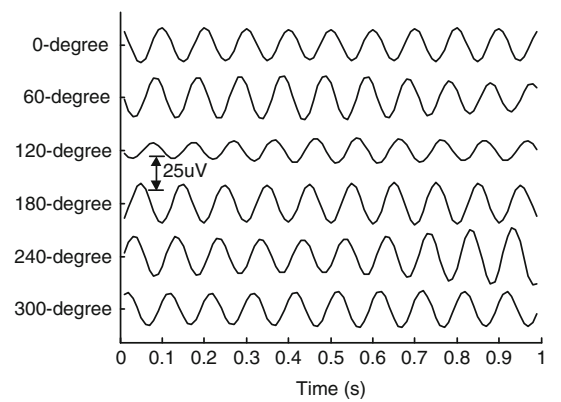

(a)

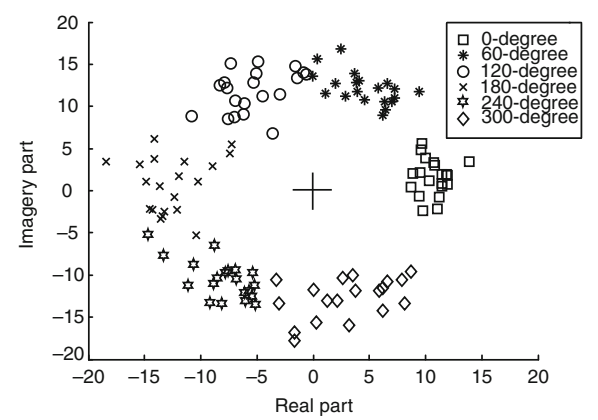

(b)

Fig. 4 (a) Examples of six phase-coded SSVEP waveforms over the occipital region. The stimulation frequency is $10 \mathrm{~Hz}$. Data were preprocessed through narrow-band filtering at 9-11 Hz. (b) Scatter diagram of complex spectrum values at the stimulation frequency. Horizontal and vertical axes correspond to real and imaginary parts of the spectrum values. The cross indicates the origin of the coordinate system

phase differences are required. For example, a computer screen refreshing signal $(60 \mathrm{~Hz})$ can be used as a basic clock to produce stable $10 \mathrm{~Hz}$ signals. In our phase modulation-based BCI, six visual targets flickering at the same frequency of $10 \mathrm{~Hz}$ but with different phases appear on the screen. The flashing moments of the visual targets are staggered by one refreshing period of the screen $(1 / 60 \mathrm{~s})$, and thus produce a phase difference of 60 degrees between two adjacent stimuli (taking six refreshing periods as 360 degrees). During the experiment, the subject was asked to gaze at the six targets, respectively, and the SSVEP was gathered from electrodes located in the occipital region. The initial phases $\phi_{0 i}(t)$ of the SSVEPs can be obtained through calculating the angle of the spectrum value at the characteristic frequency simply by the following formula:

$$
\phi_{0 i}=\operatorname{angle}\left[\frac{1}{K} \sum_{n=1}^{K} s_{i}(n) e^{-j 2 \pi\left(f_{0} / f_{s}\right) n}\right], i=1,2, \ldots, N
$$

where $f_{\mathrm{s}}$ is the sampling frequency, $f_{0}$ is the stimulation frequency, and $K$ is the data length (the number of samples). The six-target phase-coding SSVEP system was tested with a volunteer. The results are shown in Fig. 4. It is clearly shown that the SSVEPs and the stimulating signals are stably phase locked. The responses evoked by stimulating signals with a phase difference of 60 degrees also have a phase difference of approximately 60 degrees.

\section{Designs of Practical BCIs}

The principles of BCIs based on the modulation of EEG rhythms have been systematically described above. Toward the aim of practical applications, we have 
made great efforts in facilitating system configuration and improving system performance. According to the practicality issues described in the first section, we focus on the two aspects: parameter optimization and information processing. These designs can significantly reduce system cost and improve system performance. In the present BCI systems, reducing the number of electrodes is important for developing clinical applications of BCI. Therefore, design of electrode layout is a common problem for all the BCI systems. In our studies, the method of bipolar lead was employed. To ensure a stable system performance, appropriate approaches to information processing play important roles. Frequency features of SSVEP harmonics have been investigated in our designs for a frequency-coded system and have shown an increased performance. In the motor imagery BCI, phase synchrony measurement has been employed, providing information in addition to the power feature.

\subsection{Designs of a Practical SSVEP-based BCI}

The SSVEP BCI based on frequency coding seems to be rather simple in principle, but a number of problems have to be solved during its implementation. Among them, lead position, stimulation frequency, and frequency feature extraction are most important. Due to differences in the subjects' physiological conditions, a preliminary experiment was designed to be carried out for a new user to set the subject-specific optimal parameters. The practicability of the system has been demonstrated by tests in many normal subjects and some patients with spinal cord injury (SCI).

\subsubsection{Lead Position}

The goal of lead selection is to achieve SSVEPs with a high SNR using the fewest number of electrodes. Only one bipolar lead is chosen as input in our system. The procedure includes two steps: finding a signal electrode and a reference electrode. From physiological knowledge, the electrode giving the strongest SSVEP, which is generally located in the occipital region, is selected as the signal electrode. The reference electrode is searched under the following considerations: its amplitude of the SSVEP should be lower, and its position should lie in the vicinity of the signal electrode so that its noise component is similar to that of the signal electrode. A high SNR can then be gained when the potentials of the two electrodes are subtracted, producing a bipolar signal. Most of the spontaneous background activities are eliminated after the subtraction, while the SSVEP component is retained. Details of this method can be found in [21]. According to our experience, although the selection varies between subjects, once it is selected, it is relatively stable over a period of time. This finding makes this method feasible for practical BCI application. For a new subject, the multi-channel recording only needs to be done once for optimization of the lead position. 


\subsubsection{Stimulation Frequency}

Three problems related to stimulation frequency must be considered carefully. The first one concerns false positives. Generally, the SSVEPs in the alpha region $(8-13 \mathrm{~Hz})$ have high amplitudes, which can facilitate frequency detection. However, if the stimulation frequency band overlaps with alpha rhythms, the spontaneous EEG may be likely to satisfy the criteria of peak detection even though the user has not performed any intentional action. To implement an asynchronous BCI that allows the user to operate the system at any moment, avoidance of false positive is absolutely necessary. The second problem is about the efficiency of frequency detection. The criteria for confirming a stimulus frequency is the SNR threshold (SNR is defined as the ratio of EEG power at the stimulation frequency to the mean power of the adjacent frequency bands). For most subjects, background components in the SSVEP are depressed, while the signal amplitude at the stimulus frequency increases enormously. However, for some subjects, the majority of signal energy still lies within the region of background alpha activities. In these circumstances, although the stimulus frequency can be clearly identified, the SNR cannot reach the threshold predefined, and thus the decision of a command can not be made. Due to these reasons, some frequency components in the alpha region should be excluded to avoid the interference of background alpha rhythms effectively. The third problem concerns the bandwidth of usable SSVEPs. Increasing the number of visual targets is an effective approach to increase the ITR. It can be realized through extending the stimulation frequency bandwidth. In our previous study, we demonstrated that the high-frequency SSVEP $(>20 \mathrm{~Hz})$ has an SNR similar to the low-frequency SSVEP [22]. By extending stimulation frequency to a wider range, a system with more options can be designed and a higher performance can be expected.

\subsubsection{Frequency Feature}

Due to the nonlinearity during information transfer in the visual system, strong harmonics may often be found in the SSVEPs. For example, in Fig. 5, the SSVEPs elicited by the 9 and $10 \mathrm{~Hz}$ stimulations show characteristic frequency components with peaks at the fundamental and the second harmonic frequencies $(18$ and $20 \mathrm{~Hz}$, respectively). Müller-Putz et al. investigated the impact of using SSVEP harmonics on the classification result of a four-class SSVEP-based BCI [23]. In their study, the accuracy obtained with combined harmonics (up to the third harmonic) was significantly higher than with only the first harmonic. In our experience, for some subjects, the intensity of the second harmonic may sometimes be even stronger than that of the fundamental component. Thus, analysis of the frequency band should cover the second harmonic and the frequency feature has to be taken as the weighted sum of their powers, namely:

$$
P_{i}=\alpha P_{f_{1 i}}+(1-\alpha) P_{f_{2 i}}, i=1,2, \ldots, N
$$


Fig. 5 Power spectra of SSVEPs at the $\mathrm{O} 2$ electrode for one subject. The stimulation frequencies were 9 and $10 \mathrm{~Hz}$. Length of the data used for calculating the PSD is $4 \mathrm{~s}$. The spectra show clear peaks at the fundamental and the 2nd harmonic frequencies

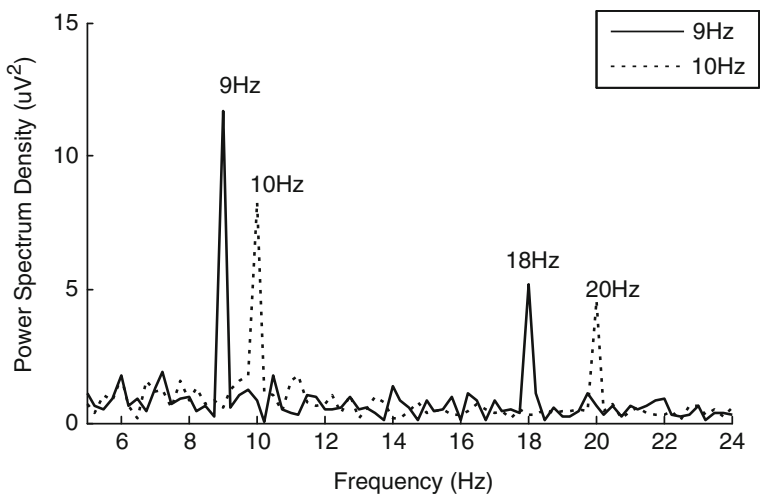

where $N$ is the number of targets and $P_{f_{1 i}}, P_{f_{2 i}}$ are respectively the spectrum peak values of fundamental and second harmonics of the $i$ th frequency (i.e., $i$ th target) and $\alpha$ is the optimized weighting factor that varies between subjects. Its empirical value may be taken as:

$$
\alpha=\frac{1}{N} \sum_{i=1}^{N} P_{f_{1 i}} /\left(P_{f_{1 i}}+P_{f_{2 i}}\right) .
$$

\subsection{Designs of a Practical Motor Imagery Based BCI}

In the study of EEG-based BCI, the system based on imagery movement is another active theme due to its relatively robust performance for communication and intrinsic neurophysiological significance for studying the mechanism of motor imagery [11]. Moreover, it is a totally independent BCI system which is likely to be more useful for completely paralyzed patients than the SSVEP-based BCI. Most of the current motor imagery based BCIs are based on characteristic ERD/ERS spatial distributions corresponding to different motor imagery states. Figure 6 displays characteristic mappings of ERD/ERS for one subject corresponding to three motor imagery states, i.e., imagining movements of left/right hands and foot. Due to the widespread distribution of ERD/ERS, techniques of spatial filtering, e.g, common spatial pattern (CSP), were widely used to obtain a stable system performance. However, due to the limit of electrodes in a practical system, electrode layout has to be carefully considered. With only a small number of electrodes, searching for new features using new information processing methods will contribute significantly to classifying motor imagery states. 

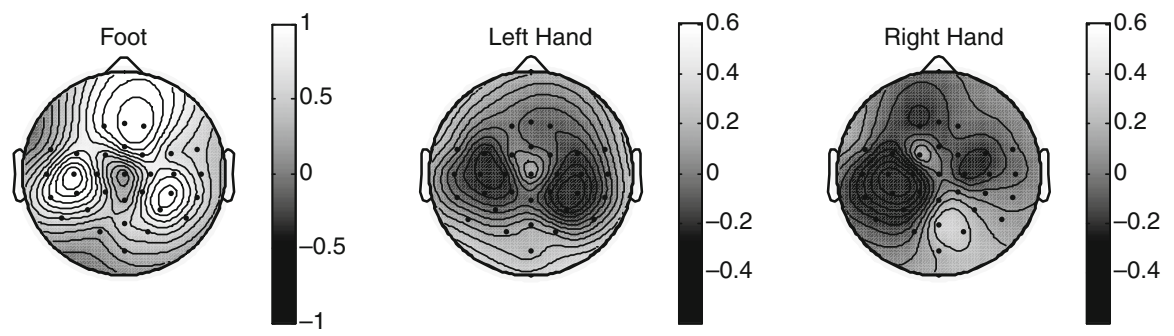

Fig. 6 Mappings of ERD/ERS of mu rhythms during motor imagery. ERD over hand areas has a distribution with contralateral dominance during hand movement imagination. During foot movement imagination, an obvious ERS appears in central and frontal areas

\subsubsection{Phase Synchrony Measurement}

In recent years, measurement of phase coupling (or phase locking) of EEG or magnetoencephalogram (MEG) has been used for exploring the dynamics of brain networking [24]. The phase-locking value (PLV) measurement has been introduced recently to extract EEG features in BCI research [25, 26].

Given $s_{\mathrm{x}}(t)$ and $s_{\mathrm{y}}(t)$ as the signals at electrodes $\mathrm{x}$ and $\mathrm{y}$, and $\phi_{\mathrm{x}}(t)$ and $\phi_{\mathrm{y}}(t)$ as their corresponding instantaneous phases, the instantaneous phase difference between the two signals is defined as $\Delta \phi(t) . \Delta \phi(t)$ is a constant when the two signals are perfectly synchronized. In scalp EEG signals with low SNR, the true synchrony is always buried in a considerable background noise; therefore, a statistical criterion has to be provided to quantify the degree of phase locking [24]. A single-trial phase-locking value is defined for each individual trial as:

$$
\operatorname{PLV}=\left|\left\langle e^{j \Delta \phi(t)}\right\rangle_{t}\right|
$$

where $\langle\cdot\rangle_{t}$ is the operator of averaging over time. In the case of completely synchronized signals, $\Delta \phi(t)$ is a constant and PLV is equal to 1 . If the signals are unsynchronized, $\Delta \phi(t)$ follows a uniform distribution and PLV approaches 0 .

Since supplementary motor area (SMA) and primary motor cortex (M1) areas are considered primary cortical regions involved in the task of motor imagery, we investigated EEG synchrony between these regions (i.e., electrode pairs of FCzC3, FCz-C4, and C3-C4 shown in Fig. 7). The statistical PLV obtained through averaging over all trials in each class presents a contralateral dominance during hand movement imagery, e.g., PLV of C3-FCz has a higher value during right-hand imagery than that of the left hand. In contrast to C3-FCz and C4-FCz, PLV shows a low synchrony level between $\mathrm{C} 3$ and $\mathrm{C} 4$ and there exists no significant difference between left- and right-hand imagery. Power features derived from ERD/ERS indicate the brain activities focused on both M1 areas, while synchronization features introduce additional information from the SMA areas. Compared with detecting the power change, the synchrony measure was proved effective to supply additional information of the brain activities in the motor cortex. Therefore, the combination of power and synchrony features is theoretically expected to improve the performance 
Fig. 7 Placement of electrodes in the motor imagery based BCI. Electrodes $\mathrm{C} 3$ and $\mathrm{C} 4$ represent lateral hand areas in $\mathrm{M} 1$, and electrode $\mathrm{FCz}$ indicates the SMA.

Anatomical regions of the SMA and M1 areas can be found in [28]

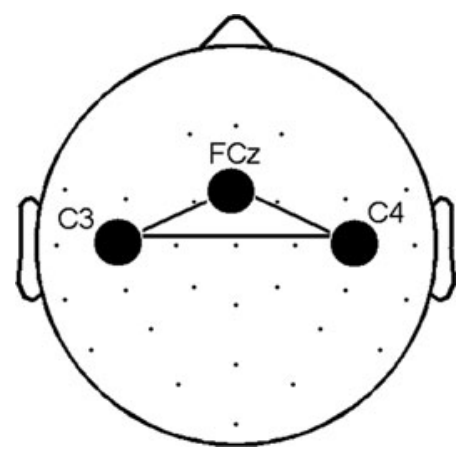

Table 1 Accuracy ( \pm standard deviation, \%) of classifying single-trial EEG during imagining movements of left/right hands and foot. The dataset of each subject consists of 360 trials (120 trials per class). Three electrodes, i.e. $\mathrm{C} 3 / \mathrm{C} 4$ and $\mathrm{FCz}$, were used for feature extraction

\begin{tabular}{llll}
\hline Subject & Synchrony & Power & Synchrony+Power \\
\hline S1 & $87.47 \pm 2.19$ & $84.83 \pm 2.47$ & $91.50 \pm 1.98$ \\
S2 & $83.25 \pm 1.78$ & $86.64 \pm 1.97$ & $90.60 \pm 1.79$ \\
S3 & $82.08 \pm 2.24$ & $80.64 \pm 2.44$ & $87.19 \pm 2.12$ \\
Mean & 84.27 & 84.04 & 89.76 \\
\hline
\end{tabular}

of classification. As illustrated in Table 1, the averaged accuracy derived from the synchrony and the power features was 84.27 and $84.04 \%$, respectively, on three subjects (10-fold cross-validation with 120 trials per class for each subject). Feature combination led to an improved performance of $89.76 \%$. The synchrony feature vector consists of two PLVs of two electrode pairs, i.e., FCz-C3, FCz-C4. The power features are the band-pass power on $\mathrm{C} 3$ and $\mathrm{C} 4$ electrodes. A subject-specific bandpass filter was used to preprocess the EEG data in order to focus on the mu rhythm. PLV and power features were calculated using a time window corresponding to the motor imagery period ( $0.5-6 \mathrm{~s}$ after a visual cue). More details about the experiment paradigm can be found in [27]. For feature combination, classification is applied to the concatenation of the power features and the synchrony features. Linear discriminant analysis (LDA) was used as the classifier. The multi-class classifier was designed in a one-versus-one manner.

\subsubsection{Electrode Layout}

Since the SMA can be considered zero-phase synchronized with the M1 area displaying ERD, the power difference between M1 areas can be more significant if using $\mathrm{FCz}$ as the reference electrode [29]. For example, during left-hand imagination, the subtraction of zero-phase synchronized $S_{\mathrm{FCz}}(t)$ from $S_{\mathrm{C} 4}(t)$ results in a much lower power, whereas the power of $S_{\mathrm{C} 3}(t)$ changes slightly after the subtraction $\left(S_{\mathrm{C} 3}(t), S_{\mathrm{C} 4}(t)\right.$, and $S_{\mathrm{FCZ}}(t)$ are ear-referenced EEG signals on $\mathrm{C} 3 / \mathrm{C} 4$ and $\mathrm{FCz}$, between which the power difference is not very significant). This idea can 
be summarized as the following inequality:

$$
\left\langle\left|S_{\mathrm{C} 4}(t)-S_{\mathrm{FCZ}}(t)\right|^{2}\right\rangle_{t}<\left\langle\left|S_{\mathrm{C} 4}(t)\right|^{2}\right\rangle_{t}<\left\langle\left|S_{\mathrm{C} 3}(t)\right|^{2}\right\rangle_{t} \approx\left\langle\left|S_{\mathrm{C} 3}(t)-S_{\mathrm{FCZ}}(t)\right|^{2}\right\rangle_{t}
$$

where $\langle\cdot\rangle_{t}$ is the operator of averaging over the left-hand imagination period, and the power difference between $S_{\mathrm{C} 3}(t)$ and $S_{\mathrm{C} 4}(t)$ is due to ERD. Therefore, the midline $\mathrm{FCz}$ electrode is a good reference to extract the power difference between left and right hemispheres, because it contains additional information derived from brain synchronization.

Compared with the approach of concatenating power and synchrony features, this bipolar approach has the advantages of a lower dimension of the features and smaller computational cost. A lower feature dimension can obtain a better generalization ability of the classifier, and smaller computational cost can assure the real-time processing. In the online system, the bipolar approach (i.e. FCz-C3 and FCz-C4) was employed to provide integrated power and synchrony information over the motor cortex areas. Considering the necessity of a practical BCI (e.g., easy electrode preparation, high performance, and low cost), electrode placement with an implicit embedding method is an efficient approach to implement a practical motor imagery based BCI. Recently, a method for optimizing subject specific bipolar electrodes was proposed and demonstrated in [30].

\section{Potential Applications}

\subsection{Communication and Control}

BCI research aims to provide a new channel for the motion disabled to communicate with the environment. Up to now, although clinical applications have been involved in some studies, most BCIs have been tested in the laboratory with normal subjects [31]. Practical designs of BCIs proposed in this chapter will benefit spreading reallife applications for the patients. For the BCI community, further investigation of clinical applications should be emphasized. In our studies, online demonstrations have been designed to fulfill some real-life applications. Due to its advantage of a high ITR, the SSVEP BCI has been employed in various applications, e.g., spelling, cursor control, and appliance control. Moreover, an environmental controller has been tested in patients with spinal cord injury [21]. Figure 8 presents the scene of using the SSVEP-based BCI to make a phone call [32]. The system consists of a small EEG recording system, an LED stimulator box including a 12-target number pad and a digitron display, and a laptop for signal processing and command transmission. A simple head-strap with two embedded electrodes was used to record data on one bipolar channel. The user could input a number easily through directing his or her gaze on the target number, and then fulfill the task of making a phone call conveniently with the laptop modem. 


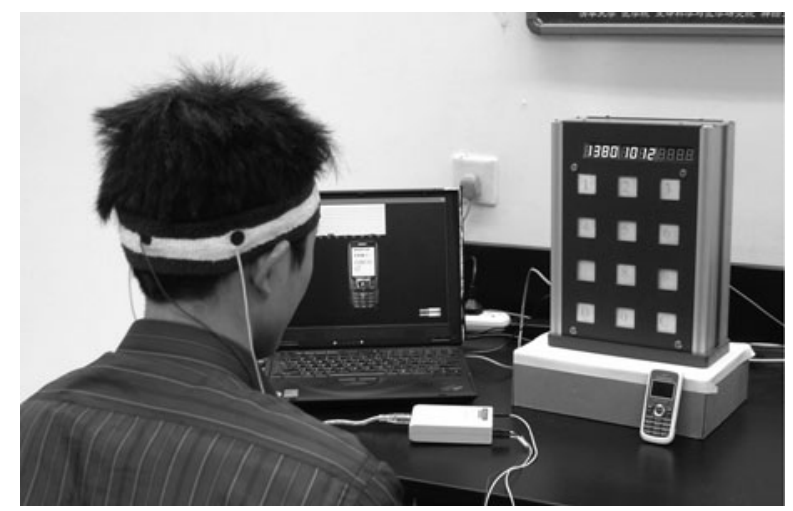

Fig. 8 Application of the SSVEP-based BCI for making a phone call. The system consists of two-lead EEG recording hardware, an LED visual stimulator, and a laptop

\subsection{Rehabilitation Training}

In addition to applications for communication and control technologies, a new area of BCI application has emerged in rehabilitation research [33] (see also chapter "Brain-Computer Interface in Neurorehabilitation" in this book). It has been proposed that BCIs may have value in neurorehabilitation by reinforcing the use of damaged neural pathways [34]. For example, some studies have demonstrated that motor imagery can help to restore motor function for stroke patients [35-37]. Based on these findings, positive rehabilitation training directed by motor intention should have a better efficiency than conventional passive training. Figure 9 shows such a device used for lower limb rehabilitation. Imagination of foot movement will start the device, while a resting state without foot movement imagination will stop the training. This system still needs further clinical investigation in the future to confirm its superiority.

Fig. 9 A system used for lower limb rehabilitation. The motor imagery based $\mathrm{BCI}$ is used to control the device. Positive rehabilitation training is performed in accordance with the concurrent motor imagery of foot movement

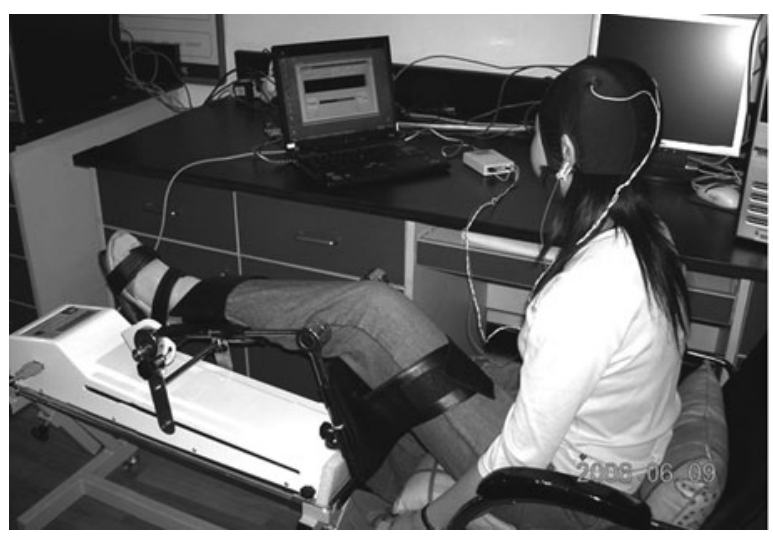




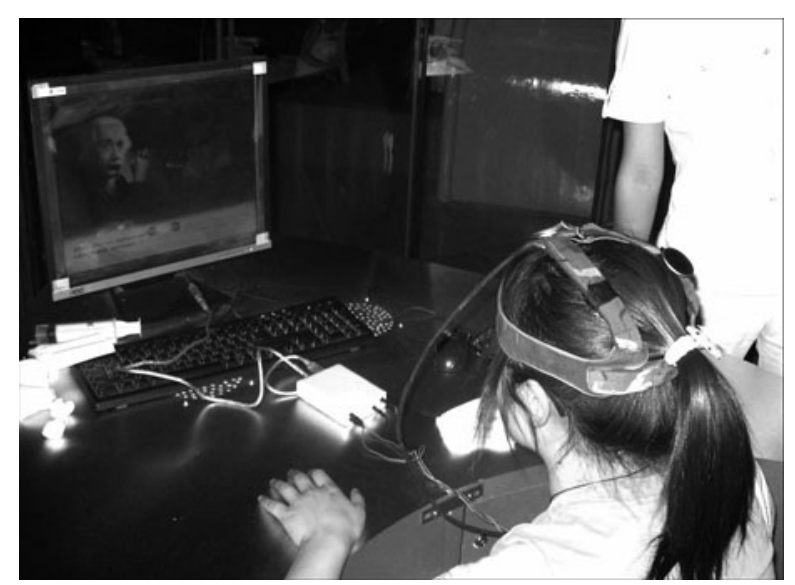

Fig. 10 A player is playing a computer game controlled through a motor imagery based BCI. This system is available for visitors to the Zhengzhou Science and Technology Center in China

\subsection{Computer Games}

BCI technology was first proposed to benefit users belonging to the disabled persons' community. However, it also has potential applications for healthy users. With numerous players, computer gaming is a potential area to employ the BCI technique $[38,39]$. Through integrating BCI to achieve additional mental control, a computer game will be more appealing. Recently, BCI technology has been used in the electronic games industry to make it possible for games to be controlled and influenced by the player's mind, e.g., Emotiv and NeuroSky systems [40, 41]. In Fig. 10, a player is absorbed in playing a game using a portable motor imagery based BCI developed in our lab. In the near future, besides the gaming industry, applications of BCI will also span various potential industries, such as neuroeconomics research and neurofeedback therapy.

\section{Conclusion}

Most of current BCI studies are still at the stage of laboratory demonstrations. Here we described the challenges in changing BCIs from demos to practically applicable systems. Our work on designs and implementations of the BCIs based on the modulation of EEG rhythms showed that by adequately considering parameter optimization and information processing, system cost could be greatly decreased while system usability could be improved at the same time. These efforts will benefit future development of BCI products with potential applications in various fields.

Acknowledgments This project is supported by the National Natural Science Foundation of China (30630022) and the Science and Technology Ministry of China under Grant 2006BAI03A17. 


\section{References}

1. J.R. Wolpaw, N. Birbaumer, D.J. McFarland, G. Pfurtscheller, and T.M. Vaughan, Braincomputer interfaces for communication and control. Clin Neurophysiol, 113, 6, 767-791, (2002).

2. M.A. Lebedev and M.A.L. Nicolelis, Brain-machine interfaces: past, present and future. Trends Neurosci, 29(9), 536-546, (2006).

3. N. Birbaumer, Brain-computer-interface research: Coming of age. Clin Neurophysiol, 117(3), 479-483, (2006).

4. G. Pfurtscheller and C. Neuper, Motor imagery and direct brain-computer communication. Proc IEEE, 89(7), 1123-1134, (2001).

5. T. Kluge and M. Hartmann, Phase coherent detection of steady-state evoked potentials: experimental results and application to brain-computer interfaces. Proceedings of 3rd International IEEE EMBS Neural Engineering Conference, Kohala Coast, Hawaii, USA, pp. 425-429, 2-5 May, (2007).

6. S. Makeig, M. Westerfield, T.P. Jung, S. Enghoff, J. Townsend, E. Courchesne, and T.J. Sejnowski, Dynamic brain sources of visual evoked responses. Science, 295(5555), 690-694, (2002).

7. E. Niedermeyer and F.H. Lopes da Silva, Electroencephalography: Basic principles, clinical applications and related fields, Williams and Wilkins, Baltimore, MD, (1999).

8. Y. Wang, X. Gao, B. Hong, C. Jia, and S. Gao, Brain-computer interfaces based on visual evoked potentials: Feasibility of practical system designs. IEEE EMB Mag, 27(5), 64-71, (2008).

9. A.C. Metting VanRijn, A.P. Kuiper, T.E. Dankers, and C.A. Grimbergen, Low-cost active electrode improves the resolution in biopotential recordings. Proceedings of 18th International IEEE EMBS Conference, Amsterdam, Netherlands, pp. 101-102, 31 Oct-3 Nov, (1996).

10. J.R. Wolpaw and D.J. McFarland, Control of a two-dimensional movement signal by a noninvasive brain-computer interface in humans. Proc Natl Acad Sci USA, 101(51), 17849-17854, (2004).

11. B. Blankertz, K.R. Muller, D.J. Krusienski, G. Schalk, J.R. Wolpaw, A. Schlogl, G. Pfurtscheller, J.D.R. Millan, M. Schroder, and N. Birbaumer, The BCI competition III: Validating alternative approaches to actual BCI problems. IEEE Trans Neural Syst Rehab Eng, 14(2), 153-159, (2006).

12. G. Pfurtscheller and F.H. Lopes da Silva, Event-related EEG/MEG synchronization and desynchronization: basic principles., Clin Neurophysiol, 110(11), 1842-1857, (1999).

13. D.J. McFarland, C.W. Anderson, K.R. Muller, A. Schlogl, and D.J. Krusienski, BCI Meeting 2005 - Workshop on BCI signal processing: Feature extraction and translation. IEEE Trans Neural Syst Rehab Eng, 14(2), 135-138, (2006).

14. J.J. Vidal, Real-time detection of brain events in EEG. Proc. IEEE, 65(5), 633-641, (1977).

15. E.E. Sutter, The brain response interface: communication through visually-induced electrical brain response. J Microcomput Appl, 15(1), 31-45, (1992).

16. M. Middendorf, G. McMillan, G. Calhoun, and K.S. Jones, Brain-computer interfaces based on the steady-state visual-evoked response. IEEE Trans Rehabil Eng, 8(2), 211-214, (2000).

17. M. Cheng, X.R. Gao, S.G. Gao, and D.F. Xu, Design and implementation of a brain-computer interface with high transfer rates. IEEE Trans Biomed Eng, 49(10), 1181-1186, (2002).

18. X. Gao, D. Xu, M. Cheng, and S. Gao, A BCI-based environmental controller for the motiondisabled. IEEE Trans Neural Syst Rehabil Eng, 11(2), 137-140, (2003).

19. B. Allison, D. McFarland, G. Schalk, S. Zheng, M. Jackson, and J. Wolpaw, Towards an independent brain-computer interface using steady state visual evoked potentials. Clin Neurophysiol, 119(2), 399-408, (2007).

20. F. Guo, B. Hong, X. Gao, and S. Gao, A brain-computer interface using motion-onset visual evoked potential. J Neural Eng, 5(4), 477-485, (2008).

21. Y. Wang, R. Wang, X. Gao, B. Hong, and S. Gao, A practical VEP-based brain-computer interface. IEEE Trans Neural Syst Rehabil Eng, 14(2), 234-239, (2006). 
22. Y. Wang, R. Wang, X. Gao, and S. Gao, Brain-computer interface based on the high frequency steady-state visual evoked potential. Proceedings of 1st International NIC Conference, Wuhan, China, pp. 37-39, 26-28 May, (2005).

23. G.R. Müller-Putz, R. Scherer, C. Brauneis, and C. Pfurtscheller, Steady-state visual evoked potential (SSVEP)-based communication: impact of harmonic frequency components. J Neural Eng, 2(4), 123-130, (2005).

24. J.P. Lachaux, E. Rodriguez, J. Martinerie, and F.J. Varela, Measuring phase synchrony in brain signals. Hum Brain Mapp, 8(4), 194-208, (1999).

25. E. Gysels and P. Celka, Phase synchronization for the recognition of mental tasks in a braincomputer interface. IEEE Trans Neural Syst Rehabil Eng, 12(4), 406-415, (2004).

26. Y. Wang, B. Hong, X. Gao, and S. Gao, Phase synchrony measurement in motor cortex for classifying single-trial EEG during motor imagery. Proceedings of 28th International IEEE EMBS Conference, New York, USA, pp. 75-78, 30 Aug-3 Sept, (2006).

27. Y. Wang, B. Hong, X. Gao, and S. Gao, Implementation of a brain-computer interface based on three states of motor imagery. Proceedings of 29th International IEEE EMBS Conference, Lyon, France, pp. 5059-5062, 23-26 Aug, (2007).

28. M.F. Bear, B.W. Connors, and M.A. Paradiso, Neuroscience: exploring the brain, Lippincott Williams and Wilkins, Baltimore, MD, (2001).

29. Y. Wang, B. Hong, X. Gao, and S. Gao, Design of electrode layout for motor imagery based brain-computer interface. Electron Lett, 43(10), 557-558, (2007).

30. B. Lou, B. Hong, X. Gao, and S. Gao, Bipolar electrode selection for a motor imagery based brain-computer interface. J Neural Eng, 5(3), 342-349, (2008).

31. S.G. Mason, A. Bashashati, M. Fatourechi, K.F. Navarro, and G.E. Birch, A comprehensive survey of brain interface technology designs. Ann Biomed Eng, 35(2), 137-169, (2007).

32. C. Jia, H. Xu, B. Hong, X. Gao, and S. Gao, A human computer interface using SSVEP-based BCI technology. Lect Notes Comput Sci, 4565, 113-119, (2007).

33. E. Buch, C. Weber, L.G. Cohen, C. Braun, M.A. Dimyan, T. Ard, J. Mellinger, A. Caria, S. Soekadar, A, Fourkas, and N. Birbaumer, Think to move: a neuromagnetic brain-computer interface (BCI) system for chronic stroke. Stroke, 39(3), 910-917, (2008).

34. A. Kubler, V.K. Mushahwar, L.R. Hochberg, and J.P. Donoghue, BCI Meeting 2005 Workshop on clinical issues and applications. IEEE Trans Neural Syst Rehabil Eng, 14(2), 131-134, (2006).

35. S. de Vries and T. Mulder, Motor imagery and stroke rehabilitation: A critical discussion. J Rehabil Med, 39(1), 5-13, (2007).

36. D. Ertelt, S. Small, A. Solodkin, C. Dettmers, A. McNamara, F. Binkofski, and G. Buccino, Action observation has a positive impact on rehabilitation of motor deficits after stroke. NeuroImage, 36(suppl 2), T164-T173, (2007).

37. M. Iacoboni and J.C. Mazziotta, Mirror neuron system: Basic findings and clinical applications. Ann Neurol, 62(3), 213-218, (2007).

38. J.A. Pineda, D.S. Silverman, A. Vankov, and J. Hestenes, Learning to control brain rhythms: making a brain-computer interface possible. IEEE Trans Neural Syst Rehabil Eng, 11(2), 181-184, (2003).

39. E.C. Lalor, S.P. Kelly, C. Finucane, R. Burke, R. Smith, R.B. Reilly, and G. McDarby, Steadystate VEP-based brain-computer interface control in an immersive 3D gaming environment. EURASIP J Appl Signal Process, 19, 3156-3164, (2005).

40. Emotiv headset (2010). Emotiv - brain computer interface technology. Website of Emotiv Systems Inc., http://www.emotiv.com. Accessed 14 Sep 2010.

41. NeuroSky mindset (2010). Website of Neurosky Inc., http://www.neurosky.com. Accessed 14 Sep 2010. 